03

\title{
Неизотермическое течение газа в эллиптическом канале с внутренним круговым цилиндрическим элементом в свободномолекулярном режиме
}

\author{
(C) О.В. Гермидер, В.Н. Попов
}

Северный (Арктический) фредеральный университет им. М.В. Ломоносова, 163002 Архангельск, Россия

e-mail: v.popov@narfu.ru

(Поступило в Редакцию 2 марта 2018 г.)

В рамках кинетического подхода в свободномолекулярном режиме найдено решение линеаризованной задачи о вычислении потока массы разреженного газа в длинном эллиптическом канале с внутренним круговым цилиндрическим элементом. Течение газа в канале обусловлено перепадами температуры и давления на концах канала. В качестве основного уравнения используется кинетическое уравнение Больцмана для бесстолкновительного газа, а в качестве граничного условия - модель диффузного отражения. Получено распределение массовой скорости газа по поперечному сечению канала. Вычислена величина приведенного потока массы газа в канале в зависимости от перепадов давления и температуры на его концах. Проведенный анализ результатов показал существенную зависимость потока массы от радиуса кругового цилиндра.

DOI: 10.21883/JTF.2019.01.46957.96-18

\section{Введение}

В последнее время значительно возрос интерес к неизотермическим течениям разреженного газа в каналах [1-19]. Интерес обусловлен фундаментальным и прикладным значениями наноразмерных систем и вакуумных технологий в различных областях химии и физики, в частности возможностью создания высокоскоростных молекулярных пучков импульсного типа [1], травлением кремневых пластин [2], оценкой утечки газа через компрессорные клапаны [3], моделированием потока газа в наноподшипниках [3] и т. Д. Для разработки и практического применения описанных выше технологий необходимо подробное описание потоков массы газа и тепла через каналы различного поперечного сечения. С учетом этого рядом авторов рассматривались каналы с различной конфигурацией: прямоугольный [3-7], эллиптический [8-11], цилиндрический [12-14], треугольный [2], в форме трапеции [15]. В работах [8-10] для получения макропараметров внутренних течений в эллиптическом цилиндре была использована $S$-модель кинетического уравнения, в [11] - уравнение Вильямса. В большинстве упомянутых выше работ для решения задач были использованы численные методы. Аналитически для каналов со сложной геометрией сечения в свободномолекулярном режиме решение линеаризованной задачи было получено для эллиптического цилиндра [8] и [16], двух коаксиальных цилиндров [17], прямоугольного канала с внутренним цилиндрическим элементом [18]. В качестве граничного условия на стенках канала в [1-4,6-13,15-18] была использована модель диффузного отражения, в [14] - модель зеркально-диффузного отражения. В связи с актуальностью исследований по неизотермическим течениям возникает необходимость получения аналитических решений в каналах со сложной геометрией сечения. В представленной работе в свободномолекулярном режиме задача массопереноса рассматривается в длинном эллиптическом канале с внутренним круговым цилиндрическим элементом. Течение газа в канале обусловлено перепадами температуры и давления на концах канала. Предполагается, что отражение молекул от стенок канала носит диффузный характер. Распределение массовой скорости газа по поперечному сечению канала находится в зависимости от отношения полуосей эллипса и радиуса внутреннего цилиндра. Величина приведенного потока массы газа в канале рассчитывается в зависимости от перепадов давления и температуры на его концах.

\section{1. Постановка задачи. Кинетическое уравнение}

Рассмотрим течение разреженного газа между двумя резервуарами, соединяющимися эллиптическим каналом

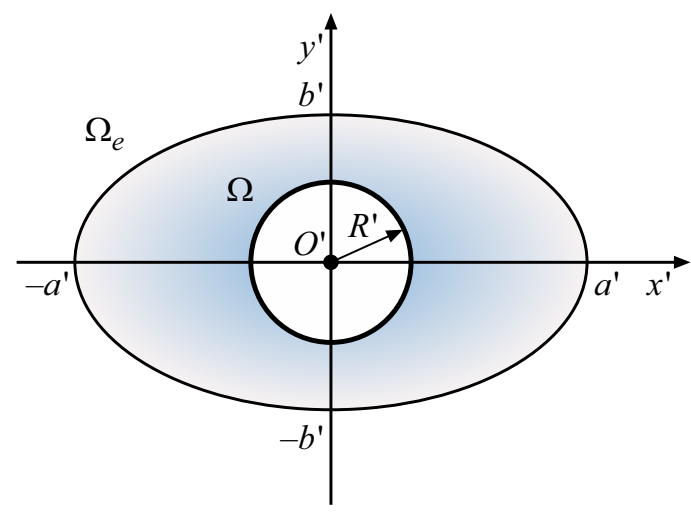


с внутренним круговым цилиндрическим элементом радиусом $R^{\prime}$. Давление и температура в первом и во втором резервуарах остаются постоянными и равными соответственно $p_{1}, T_{1}$ и $p_{2}, T_{2}$, причем $p_{2}>p_{1}$ и $T_{2}>T_{1}$. Начало декартовой системы координат выбрано в сечении, расположенном в середине канала. Будем рассматривать течение газа в средней части канала, в окрестности точки $z^{\prime}=0$. Введем обозначения для границ сечения канала: $\Omega$ - окружность радиусом $R^{\prime}$; $\Omega_{e}$ - эллипс с полуосями $a^{\prime}$ и $b^{\prime}\left(a^{\prime} \geq b^{\prime}\right) ; \Omega \cup \Omega_{e}$ (см. рисунок). Предположим, что длина канала $L^{\prime}>a^{\prime}$. В этом случае давление и температура газа релаксируют значительно быстрее в поперечном сечении, чем по всей длине канала [19]. Таким образом, можно предположить, что давление и температура зависят только от продольной координаты $z^{\prime}$. Будем полагать, что их безразмерные градиенты являются малыми по абсолютной величине, т. е.

$$
G_{T}=\frac{z^{\prime}}{T_{0}} \frac{d T}{d z^{l}}, \quad\left|G_{T}\right| \ll 1, \quad G_{p}=\frac{z^{l}}{p_{0}} \frac{d p}{d z^{l}}, \quad\left|G_{p}\right| \ll 1 .
$$

Выберем в качестве размерного масштаба малую полуось эллипса $b^{\prime}$. При этом безразмерные величины будем обозначать без штриха: $x=x^{\prime} / b^{\prime}, y=y^{\prime} / b^{\prime}$, $z=z^{\prime} / b^{\prime}$. В линейном приближении давление и температура газа имеют вид

$$
T(z)=T_{0}\left(1+G_{T} z\right), \quad p(z)=p_{0}\left(1+G_{p} z\right),
$$

где $p_{0}, T_{0}$ - давление и температура газа в начале координат. Состояние разреженного газа в точке, радиусвектор которой $r$ имеет координаты $x, y$ и $z$, определяем функцией распределения $f_{\Gamma}(r, v)$, где $v$ - скорость молекул газа. Сделанное предположение о длине канала позволяет пренебречь концевыми эффектами и рассмотреть только продольную составляющую массовой скорости $u_{z}(r)[9]$, которую находим согласно [20] как

$$
u(r)=\frac{1}{n(z)} \int v f(r, v) d^{3} v
$$

где $n(z)$ - концентрация молекул газа. Введем безразмерную компоненту вектора массовой скорости газа, следуя [19]

$$
U=\beta^{1 / 2} u
$$

где $\beta=m /\left(2 k_{\mathrm{B}} T_{0}\right), m-$ масса молекул газа, $k_{\mathrm{B}}-$ постоянная Больцмана. Приведенный поток массы газа через поперечное сечение определяем согласно [19] как

$$
\begin{aligned}
& J_{M}=\frac{J_{M}^{\prime}}{\pi\left(a^{\prime} b^{\prime}-R^{\prime 2}\right) p(z)} \sqrt{\frac{2 k_{\mathrm{B}} T(z)}{m}}=\frac{8\left(I_{1}+I_{2}\right)}{\pi\left(a-R^{2}\right)}, \\
& I_{1}=\int_{0}^{R} \int_{\sqrt{R^{2}-y^{2}}}^{a \sqrt{1-y^{2}}} U_{z}(r) d x d y, I_{2}=\int_{R}^{1} \int_{0}^{a \sqrt{1-y^{2}}} U_{z}(r) d x d y,
\end{aligned}
$$

где $J_{M}^{\prime}-$ размерный поток массы газа в канале. Для нахождения функции распределения молекул газа $f(r, v)$ воспользуемся кинетическим уравнением Больцмана, которое в декартовой прямоугольной системе координат в отсутствии межмолекулярных столкновений имеет вид [20]

$$
C_{x} \frac{\partial f}{\partial x}+C_{y} \frac{\partial f}{\partial y}+C_{z} \frac{\partial f}{\partial z}=0,
$$

где $C=\beta^{1 / 2} v-$ безразмерная скорость молекул газа. В качестве граничного условия на обтекаемых газом стенках канала будем использовать модель диффузного отражения. В этом случае [20]

$$
f^{+}\left(r_{\Gamma}, C\right)=f_{\Gamma}\left(r_{\Gamma}, C\right), \quad C_{n}>0 .
$$

Здесь $f^{+}\left(r_{\Gamma}, C\right)$ - функция распределения молекул газа, отраженных от стенок канала, $r_{\Gamma}-$ безразмерный радиус-вектор точек стенок канала, $n$ - вектор нормали к обтекаемой газом поверхности, направленный в сторону газа; $f_{\Gamma}\left(r_{\Gamma}, C\right)$ - локально равновесная функция распределения:

$$
f_{\Gamma}\left(r_{\Gamma}, C\right)=n_{\Gamma}(z)\left(\frac{m}{2 \pi k_{\mathrm{B}} T_{\Gamma}(z)}\right)^{3 / 2} \exp \left(-\frac{T_{0}}{T_{\Gamma}(z)} C^{2}\right)
$$

где $T_{\Gamma}(z), n_{\Gamma}(z) \quad$ - температура $\quad$ и $\quad$ концентрация молекул газа на поверхности канала. Линеаризуя (7) относительно абсолютного максвеллиана $f_{0}(C)=$ $=n_{0}(\beta / \pi)^{3 / 2} \exp \left(-C^{2}\right)$ и учитывая, что $p(z)=n(z) k_{\mathrm{B}} T$, приходим к следующему выражению для локально равновесной функции распределения:

$$
f_{\Gamma}(z, C)=f_{0}(C)\left(1+G_{T}\left(C^{2}-\frac{5}{2}\right) z+G_{p} z\right) .
$$

Функцию распределения $f(r, v)$ линеаризуем относительно $f_{\Gamma}(z, C)$. Принимая во внимание $(8)$, получим

$$
f(r, C)=f_{0}(C)\left(1+G_{T}\left(C^{2}-\frac{5}{2}\right) z+G_{p} z+C_{z} h(x, y, C)\right) .
$$

Подставляя функцию распределения (9) в (5), для определения $h(x, y, C)$ приходим к уравнению

$$
C_{x} \frac{\partial h}{\partial x}+C_{y} \frac{\partial h}{\partial y}+G_{p}+G_{T}\left(C^{2}-\frac{5}{2}\right)=0 .
$$

Осуществим переход к сферическим координатам в пространстве скоростей: $C_{x}=C \cos \varphi \sin \theta, C_{y}=$ $=C \sin \varphi \sin \theta, C_{z}=C \cos \theta$, где углы $\varphi$ и $\theta$ отсчитываются от положительных направлений осей $C_{x}$ и $C_{z}$ в пространстве скоростей соответственно. Представим функцию $h(x, y, C)$ в виде разложения по ортогональным в смысле равенства нулю скалярного произведения функций $e_{1}=1, e_{2}=1 / C-3 \sqrt{\pi} / 8$ и $e_{3}=C-5 /(2 C)$ :

$$
\begin{aligned}
& h(x, y, C)=G_{p}\left(Z_{1}(x, y, \varphi, \theta)+\left(\frac{1}{C}-\frac{3 \sqrt{\pi}}{8}\right)\right. \\
& \left.\times Z_{2}(x, y, \varphi, \theta)\right)+G_{T}\left(C-\frac{5}{2 C}\right) Z_{3}(x, y, \varphi, \theta) .
\end{aligned}
$$


Здесь скалярное произведение функций $f_{1}(C)$ и $f_{2}(C)$ определяется следующим образом [21]:

$$
\left(f_{1}, f_{2}\right)=\int_{0}^{+\infty} f_{1}(C) f_{2}(C) C^{5} \exp \left(-C^{2}\right) d C .
$$

Подставляя (11) в (10), в силу ортогональности функций $e_{1}, e_{2}$ и $e_{3}$ получаем три независимых уравнения для определения неизвестных функций $Z_{i}(x, y, \varphi, \theta)$ :

$$
\begin{gathered}
\left(\cos \varphi \frac{\partial Z_{1}}{\partial x}+\sin \varphi \frac{\partial Z_{1}}{\partial y}\right) \sin \theta+\frac{3 \sqrt{\pi}}{8}=0, \\
\left(\cos \varphi \frac{\partial Z_{j}}{\partial x}+\sin \varphi \frac{\partial Z_{j}}{\partial y}\right) \sin \theta+1=0, \quad j=2,3,
\end{gathered}
$$

с граничными условиями

$$
Z_{i}\left(x_{\Gamma}, y_{\Gamma}, \varphi, \theta\right)=0, \quad C n>0, \quad i=1-3 .
$$

Учитывая, что уравнения (13) и граничные условия (14) имеют одинаковую структуру для каждой функции $Z_{j}(x, y, \varphi, \theta)$, для краткости обозначим $Z(x, y, \varphi, \theta)=Z_{j}(x, y, \varphi, \theta), j=2,3$. Замечая при этом, что $Z_{1}=3 \sqrt{\pi} Z / 8$, получаем для ненулевой компоненты $U_{z}$ вектора $\mathbf{U}$, определяемого равенствами (2), (3), (9) и (11), следующее выражение

$$
\begin{aligned}
U_{z}(x, y) & =\pi^{-3 / 2} \int \exp \left(-C^{2}\right) C_{z}^{2} h(x, y, C) d^{3} C \\
& =G_{T} U_{z}^{(1)}(x, y)+G_{p} U_{z}^{(2)}(x, y), \\
U_{z}^{(1)}(x, y) & =-\frac{1}{4 \pi^{3 / 2}} \int_{0}^{\pi} \cos ^{2} \theta \sin \theta \int_{0}^{2 \pi} Z(x, y, \varphi, \theta) d \varphi d \theta \\
& =-\frac{U_{z}^{(2)}(x, y)}{2} .
\end{aligned}
$$

Из (15) следует, что приведенный поток массы, определяемый по формуле (4), также можно представить в виде суммы двух линейных по градиентам температуры и давления функций:

$$
\begin{gathered}
J_{M}=G_{T} J_{M}^{(1)}+G_{p} J_{M}^{(2)}, \quad J_{M}^{(i)}=\frac{8\left(I_{1}^{(i)}+I_{2}^{(i)}\right)}{\pi\left(a-R^{2}\right)}, \\
I_{1}^{(i)}=\int_{0}^{R^{a}} \int_{\sqrt{R^{2}-y^{2}}}^{a y^{2}} U_{z}^{(i)}(x, y) d x d y, \\
I_{2}^{(i)}=\int_{R}^{1} \int_{0}^{a \sqrt{1-y^{2}}} U_{z}^{(i)}(x, y) d x d y, \quad i=1,2 .
\end{gathered}
$$

Здесь коэффициенты $J_{M}^{(1)}$ и $J_{M}^{(2)}=-2 J_{M}^{(1)}$ не зависят от $G_{T}$ и $G_{p}$, а слагаемые в (17) для $J_{M}$ определяют соотвественно приведенные потоки массы разреженного газа, вызываемые градиентом температуры в задаче о тепловом крипе и градиентом давления в задаче о течении Пуазейля. Получим значения этих коэффициентов для заданной конфигурации сечения канала. Для этого найдем явный вид функции $Z(x, y, \varphi, \theta)$. В силу симметрии сечения канала относительно координатных осей в конфигурационном пространстве рассмотрим точки из первого квадранта, для которых можно выделить две области изменения угла $\varphi$ :

1. $\varphi_{0}-\xi \leq \varphi \leq \varphi_{0}+\xi-$ для молекул, которые отразились от внутренней поверхности, где углы $\varphi_{0}$ и $\xi$ определяются выражениями $\varphi_{0}=\operatorname{arctg}(y / x)$, $\xi=\arcsin \left(R / \sqrt{x^{2}+y^{2}}\right)$;

2. $\varphi_{0}+\xi \leq \varphi \leq 2 \pi+\varphi_{0}-\xi$ - для молекул, которые отразились от внешней поверхности.

В первом случае решение уравнения (13) с граничным условием (14) имеет вид [18]

$$
\begin{gathered}
Z(x, y, \varphi, \theta)=-\frac{w_{1}(x, y, \varphi)}{\sin \theta}, \\
w_{1}(x, y, \varphi)=x \cos \varphi+y \sin \varphi \\
+\sqrt{R^{2}-(y \cos \varphi-x \sin \varphi)^{2}} .
\end{gathered}
$$

Во втором случае получаем [16]

$$
\begin{gathered}
Z(x, y, \varphi, \theta)=-\frac{w_{2}(x, y, \varphi)}{\sin \theta}, \\
w_{2}(x, y, \varphi)=\frac{x \cos \varphi+a^{2} y \sin \varphi}{a^{2} \sin ^{2} \varphi+\cos ^{2} \varphi} \\
+\frac{a \sqrt{a^{2} \sin ^{2} \varphi+\cos ^{2} \varphi-(y \cos \varphi-x \sin \varphi)^{2}}}{a^{2} \sin ^{2} \varphi+\cos ^{2} \varphi} .
\end{gathered}
$$

Подставляя (18)-(21) в (16), получаем

$$
\begin{aligned}
U_{z}^{(2)}(x, y)= & -\frac{1}{4 \sqrt{\pi}}\left(2 \int_{\varphi_{0}}^{\varphi_{0}+\xi} w_{1}(x, y, \varphi) d \varphi\right. \\
& \left.+\int_{\varphi_{0}+\xi}^{2 \pi+\varphi_{0}-\xi} w_{2}(x, y, \varphi) d \varphi\right) .
\end{aligned}
$$

Результаты вычислений $-J_{M}^{(2)}$ по формуле (17) с учетом полученного выражения (22) для компоненты $U_{z}^{(2)}(x, y)$ представлены в таблице в сравнении с результатами, полученными в $[8,9]$ для эллиптического канала. Для канала, образованного двумя коаксиальными цилиндрами $(a=1)$, значения $-J_{M}^{(2)}$ из таблицы при $R=0.01$ и $R=0.1$ соответственно равны 1.4932 и 1.3890. Эти же значения были получены в [17] в пределе свободномолекулярного режима на основе $S$-модели кинетического уравнения. В работе [17] массовый поток был представлен в виде ряда по малому параметру $R$, в котором отброшены члены выше четвертого порядка малости. Как следует из приведенных в таблице значений поток массы существенно зависит от $R$. 
Значения $-J_{M}^{(2)}$ в зависимости от $a, R$

\begin{tabular}{c|c|c|c|c|c|c}
\hline \multirow{2}{*}{$a$} & \multicolumn{5}{|c}{$R$} \\
\cline { 2 - 7 } & \multicolumn{2}{|c|}{0} & 0.01 & 0.1 & 0.5 & 0.9 \\
\cline { 2 - 7 } & $(17)$ & {$[8,9]$} & $(17)$ & $(17)$ & $(17)$ & $(17)$ \\
\hline 1 & 1.5045 & 1.5045 & 1.4932 & 1.3890 & 0.8701 & 0.2218 \\
1.1 & 1.5770 & 1.5770 & 1.5657 & 1.4617 & 0.9468 & 0.3250 \\
2 & 2.0655 & 2.0655 & 2.0542 & 1.9509 & 1.4684 & 1.0174 \\
5 & 2.8888 & 2.8888 & 2.8775 & 2.7752 & 2.3312 & 2.0084 \\
10 & 3.5397 & 3.5397 & 3.5284 & 3.4265 & 2.9993 & 2.7214 \\
20 & 4.1991 & 4.1991 & 4.1878 & 4.0863 & 3.6689 & 3.4152 \\
100 & 5.7387 & 5.7390 & 5.7274 & 5.6262 & 5.2183 & 4.9868
\end{tabular}

\section{2. Неизотермическое течение разреженного газа при произвольных перепадах давления и температуры}

В эксперименте, как правило, измеряется величина массы газа в канале в зависимости от перепадов давлений и температур на его концах. В случае малых перепадов температуры и давления на концах канала, распределения температуры и давления вдоль канала можно считать линейными [19]. При этом градиенты температуры и давления могут быть определены по формулам [19]

$$
G_{T}=\frac{T_{2}-T_{1}}{L T_{a v}}, \quad G_{p}=\frac{p_{2}-p_{1}}{L p_{a v}}
$$

где $L=L^{\prime} / b^{\prime}, T_{a v}=\left(T_{2}+T_{1}\right) / 2, \quad p_{a v}=\left(p_{2}+p_{1}\right) / 2$ и перепады температуры и давления являются малыми: $\left(T_{2}-T_{1}\right) \ll T_{1}$ и $\left(p_{2}-p_{1}\right) \ll p_{1}$. В этом случае величина $J_{M}$ остается постоянной. Если отношения $T_{2} / T_{1}$ и $p_{2} / p_{1}$ являются большими, то распределение давления перестает быть линейным и происходит изменение величины $J_{M}$ вдоль канала. Так как теплопроводность стенок канала значительно больше, чем теплопроводность газа, распределение температуры, определяемое тепловыми свойствами канала, можно считать линейным [9]: $T\left(z_{*}\right)=\left(T_{2}-T_{1}\right) z_{*}+\left(T_{1}+T_{2}\right) / 2$, где $z_{*}=z^{\prime} / L^{\prime}$. Распределение давления при этом заранее неизвестно, а должно быть найдено в результате решения кинетического уравнения. Следуя $[3,9,15,19]$, определим безразмерный поток массы как

$$
J_{M}^{*}=\frac{J_{M}^{\prime} L}{\pi\left(a^{\prime} b^{\prime}-R^{\prime 2}\right) p_{1}} \sqrt{\frac{2 k_{\mathrm{B}} T_{1}}{m}},
$$

где величина $J_{M}^{*}$ остается постоянной вдоль длины канала. Для определения давления газа в канале подставляем последовательно выражения (4) и (24) в (17). В результате с учетом того, что $J_{M}^{(2)}=-2 J_{M}^{(1)}$, получаем дифференциальное уравнение относительно неизвестной функции $p\left(z^{*}\right)$ :

$$
\frac{1}{p_{1}} \frac{d p}{d z_{*}}=\frac{p\left(z_{*}\right)}{2 p_{1} T\left(z_{*}\right)} \frac{d T}{d z_{*}}+\sqrt{\frac{T\left(z_{*}\right)}{T_{1}}} \frac{J_{M}^{*}}{J_{M}^{(2)}},
$$

где $J_{M}^{*}$ является параметром, а значения $J_{M}^{(2)}$ определяются по формуле (17). С учетом линейного распределения температуры вдоль длины канала общее решение дифференциального уравнения (25) имеет вид

$$
p\left(z_{*}\right)=\frac{p_{1} T\left(z_{*}\right)^{3 / 2} J_{M}^{*}}{\left(T_{2}-T_{1}\right) J_{M}^{(2)} \sqrt{T_{1}}}+C_{1} \sqrt{T\left(z_{*}\right)},
$$

где $C_{1}-$ константа интегрирования. Подставляя в (26) граничные условия $p(-1 / 2)=p_{1}$ и $p(1 / 2)=p_{2}$, приходим к системе уравнений для определения $C_{1}$ и $J_{M}^{*}$. Введя обозначение

$$
J_{M}^{*}=\left(\frac{p_{*}}{\sqrt{T_{*}}}-1\right) J_{M}^{(2)}, \quad C_{1}=\frac{p_{1}\left(p_{*}-T_{*}^{3 / 2}\right)}{\left(1-T_{*}\right) \sqrt{T_{2}}} .
$$

Коэффициент перед $J_{M}^{(2)}$ в выражении (27) для $J_{M}^{*}$ совпадает с аналогичным результатом [19] для цилиндрического канала. Подставляя выражения (27) в (26), находим $p\left(z_{*}\right)$ :

$$
\begin{aligned}
& p\left(z_{*}\right)= \\
& =\frac{p_{1}\left(p_{*}+\sqrt{T_{*}}+2 z_{*}\left(p_{*}-\sqrt{T_{*}}\right)\right) \sqrt{2\left(T_{*}+1+2\left(T_{*}-1\right) z_{*}\right)}}{4 \sqrt{T_{*}}} .
\end{aligned}
$$

В случае изобарического течения $p_{*}=1\left(p_{1}=p_{2}\right)$ из (27) следует, что $J_{M}^{*}$ принимает только положительные значения, равные $-\left(1-1 / \sqrt{T_{*}}\right) J_{M}^{(2)}$. При выполнении этого условия имеет место явление теплового крипа, т.е. газ течет от холодного резервуара к горячему, что соответствует выводам, приведенным в [19].

Для неизобарического течения $\left(p_{*}>1\right)$ величина $J_{M}^{*}<0$, если $p_{*}>\sqrt{T_{*}}$. В этом случае имеет место течение Пуазейля и газ течет от горячего резервуара, где давление выше, к холодному, где давление ниже. При $p_{*} \leq \sqrt{T_{*}}$ величина $J_{M}^{*} \geq 0$ и имеет место явление теплового крипа.

Полученные по формуле (27) значения $J_{M}^{*}$ хорошо согласуются с результатами из [9] для эллиптических каналов $(R=0)$ для режимов, близких к свободномолекулярному. В частности, при $p_{*}=1$ и $T_{*}=3.8$ для $a=1$ и $a=10$ по формуле (27) при подстановке в нее значений из таблицы, находим $J_{M}^{*}=0.7327$ и $J_{M}^{*}=1.7239$. Соответствующие значения этой величины из [9] равны 0.7144 и 1.5916. При $p_{*}=100$ и $T_{*}=3.8$ для $a=1$ и $a=10$ значения $J_{M}^{*} \cdot 10^{-2}$, вычисленные по формуле (27), становятся отрицательными: -0.757 и -1.780 , а из [9] они равны -0.738 и -1.511 . 


\section{Заключение}

Итак, в представленной работе решена линеаризованная задача о вычислении потока массы газа в длинном эллиптическом канале с внутренним круговым цилиндрическим элементом в свободномолекулярном режиме. Приведены зависимости величины приведенного потока массы газа в канале от произвольных перепадов давления и температуры на его концах при условии, что полуоси канала много меньше его длины. Показано, что значения потока массы уменьшаются нелинейно в зависимости от радиуса внутреннего цилиндра.

Работа выполнена при финансовой поддержке РФФИ в рамках научного проекта 19-01-00506.

\section{Список литературы}

[1] Конопелько Н.А., Титарев В.А., Шахов Е.М. // Журн. вычисл. матем. и матем. физ. 2016. Т. 56. Вып. 3. С. 476-489.

[2] Naris S., Valougeorgis D. // European J. Mechanics. B: Fluids. 2008. Vol. 27. P. 810-822.

[3] Graur I., Ho M.T. // Vacuum. 2014. Vol. 101. P. 328-332.

[4] Sharipov F.M. // J. Vac. Sci. Technol. A. 1999. Vol. 17. N 5. P. 3062-3066.

[5] Pantazis S., Varoutis S., Hauer V., Day C., Valougeorgis D. // Vacuum. 2011. Vol. 85. P. 1161-1164.

[6] Титарев В.А., Шахов Е.М. // Журн. вычисл. матем. и матем. физ. 2010. Т. 50. Вып. 7. С. 1285-1302.

[7] Гермидер О.В., Попов В.Н., Юиканов А.А. // ЖТФ. 2016. Т. 86. Вып. 6. С. 37-41.

[8] Graur I., Sharipov F. // Europ. J. of Mechanics B: Fluids. 2008. Vol. 27. P. 335-345.

[9] Graur I., Sharipov F. // Microfluid Nanofluid. 2009. Vol. 6. P. 267-275.

[10] Rykov V.A., Titarev V.A., Shakhov E.M. // Fluid Dynamics. 2011. Vol. 46. № 3. P. 456-466.

[11] Гермидер О.В., Попов В.Н. // ЖТФ. 2017. Т. 87. Вып. 3. C. 331-334

[12] Kamphorst C.H., Rodrigues P., Barichello L.B. // Appl. Mathematics. 2014. Vol. 5. P. 1516-1527.

[13] Siewert C.E., Valougeorgis D. // J. Quant. Spectrosc. Radiat. Transf. 2002. Vol. 72. P. 531-550.

[14] Гермидер О.В., Попов В.Н. // ЖТФ. 2017. Т. 87. Вып. 11. C. 1603-1608.

[15] Ritos K., Lihnaropoulos Y., Naris S., Valougeorgis D. 2nd Micro and Nano Flows Conference, Brunel University, West London, UK. 2009.

[16] Гермидер О.В., Попов В.Н., Юшканов А.А. // Сибирский журнал индустриальной математики. 2017. Т. 20. Вып. 3. C. 24-30.

[17] Шахов Е.М. // Журн. вычисл. матем. и матем. физ. 2003. T. 43. Вып. 7. С. $1107-1116$.

[18] Гермидер О.В., Попов В.Н. // Сибирские электронные математические известия. 2017. Т. 14. С. 518-527.

[19] Шарипов Ф.М., Селезнев В.Д. Движение разреженных газов в каналах и микроканалах. Екатеринбург: УрО РАН, 2008. $230 \mathrm{c}$.

[20] Коган М.Н. Динамика разреженного газа. Кинетическая теория. М.: Наука, 1967. 440 с.

[21] Латышев А.В., Юшканов А.А. Кинетические уравнения типа Вильямса и их точные решения: монография. М.: МГОУ, 2004. $271 \mathrm{c}$. 